\title{
Energetic electron and ion generation from interactions of intense laser pulses with laser machined conical targets
}

\author{
T. Matsuoka ${ }^{1}$, S. Reed ${ }^{1}$, C. McGuffey ${ }^{1}$, S.S. Bulanov ${ }^{1}$, F. Dollar ${ }^{1}$, \\ L. Willingale ${ }^{1}$, V. Chvykov ${ }^{1}$, G. Kalinchenko ${ }^{1}$, A. Brantov ${ }^{2}$, \\ V. Yu. Bychenkov ${ }^{2}$, P. Rousseau ${ }^{1}$, V. Yanovsky ${ }^{1}$, D.W. Litzenberg ${ }^{3}$, \\ K. Krushelnick ${ }^{1}$ and A. Maksimchuk ${ }^{1}$ \\ ${ }^{1}$ Center for Ultrafast Optical Science, University of Michigan, Ann Arbor, MI, USA \\ ${ }^{2}$ P. N. Lebedev Physics Institute, Russian Academy of Sciences, Moscow, Russia \\ ${ }^{3}$ Department of Radiation Oncology, University of Michigan, Ann Arbor, MI, USA
}

Received 26 June 2009, accepted for publication 22 April 2010

Published 4 May 2010

Online at stacks.iop.org/NF/50/055006

\begin{abstract}
The generation of energetic electron and proton beams was studied from the interaction of high intensity laser pulses with pre-drilled conical targets. These conical targets are laser machined onto flat targets using 7-180 $\mu \mathrm{J}$ pulses whose axis of propagation is identical to that of the main high intensity pulse. This method significantly relaxes requirements for alignment of conical targets in systematic experimental investigations and also reduces the cost of target fabrication. These experiments showed that conical targets increase the electron beam charge by up to $44 \pm 18 \%$ compared with flat targets. We also found greater electron beam divergence for conical targets than for flat targets, which was due to escaping electrons from the surface of the cone wall into the surrounding solid target region. In addition, the experiments showed similar maximum proton energies for both targets since the larger electron beam divergence balances the increase in electron beam charge for conical targets. 2D particle in cell simulations were consistent with the experimental results. Simulations for conical target without preplasma showed higher energy gain for heavy ions due to 'directed coulomb explosion'. This may be useful for medical applications or for ion beam fast ignition fusion.
\end{abstract}

PACS numbers: $52.57 . \mathrm{Kk}$

(Some figures in this article are in colour only in the electronic version)

\section{Introduction}

The interaction of ultra-short laser pulses and hollow conical targets is potentially important for applications such as the fast ignition (FI) scheme for inertial fusion energy [1], high energy density physics [2], proton beam production [3,4] for hadronic medical therapy $[5,6]$, as well as $\mathrm{x}$-ray generation applications [7] - due to the higher flux and energies of the hot electrons which are generated with laser-cone interactions compared with simple flat targets [3,4]. Experiments and simulations have shown that during these interactions the laser pulse energy is absorbed at the wall of conical targets and selfgenerated magnetic fields at the surface guide the hot electrons along the wall $[8,9]$. This increases electron beam flux at the conical tip and thus also increases the energy of accelerated protons. However, recent works have revealed that conical targets actually lowered electron beam flux compared with the flat one $[10,11]$. Although the benefit of keeping open path for the heating laser is clear, more systematic research is required for a better understanding of these interactions, particularly for optimization of the geometry, such as the hole depth, opening angle and the tip thickness of the conical targets for charged particle beam production. In particular, important investigations can now be undertaken using high repetition rate laser systems rather than the larger low repetition rate laser facilities which have been used for this research previously.

There are several important outstanding issues with regard to the use of conical targets for these applications. One is the production cost of such targets-which is particularly important for eventual inertial fusion energy applications. It is even more an important consideration for fundamental experimental investigation on such complex target configurations. The use of lithographic technology for mass production of conical targets has previously been reported $[4,7]$ and may reduce such costs substantially in the future. Another issue with the use of conical targets for FI has been the alignment of the target with respect to the laser axis. This is important since a shift of the target axis in the perpendicular 
plane to the laser axis can significantly reduce the conversion efficiency from the laser pulse to hot electrons $[12,13]$. Finally an issue for applications to FI is that during ICF implosions the conical target is surrounded by dense low temperature plasma which will increase the hot electron beam divergence angle, thereby decreasing the heating efficiency. This effect has been observed previously in 2D-PIC (particle in cell) simulations [14] but has been more difficult to investigate experimentally. Previous experiments used cone targets with wall thicknesses of a few tens of micrometres with the outside of the cone in vacuum [3]. Geometries which are relevant to the 'realistic' situation in which the cone is surrounded by high density low temperature material could be achieved by using conical targets that are formed in flat targets having dimensions perpendicular to the conical axis in the millimetre range. In this paper, we report an experimental study of hot electron and $\sim \mathrm{MeV}$ proton generation using conical targets which were laser machined in situ in order to match the conical target axis and the laser axis within a spatial scale of a few micrometres.

\section{Production process of conical target and experimental configuration}

Experiments were performed using the HERCULES (Ti : sapphire) laser facility at the Center for Ultrafast Optical Science at the University of Michigan [15]. A 30 TW laser pulse at $\lambda=800 \mathrm{~nm}$ was focused by an $F / 2$ parabolic mirror having a $60^{\circ}$ off axis angle. A focal spot of $3 \mu \mathrm{m}$ full width half maximum (FWHM) was achieved using the wavefront correction method [16]. A focused peak intensity of $4 \times 10^{20} \mathrm{~W} \mathrm{~cm}^{-2}$ (corresponding to $a_{0}=14$ ) is obtained for $30 \mathrm{TW}$ laser power having a FWHM pulse duration of $\tau=30 \mathrm{fs}$. Here the normalized vector potential is given by $a_{0}=0.85 \times 10^{-9} \lambda_{\mu} \sqrt{I}$ with wavelength of the laser in micrometres $\left(\lambda_{\mu}\right)$ and laser intensity $(I)$ in units of $\mathrm{W} \mathrm{cm}^{-2}$. The standard deviation of power fluctuations was less than $\pm 10 \%$ throughout the experiments shown in this paper. In order to minimize preplasma formation, the temporal contrast of the pulse was controlled using the crossed polarized wave technique which can provide $10^{11}$ amplified spontaneous emission (ASE) contrast [17]. The contrast is defined as the intensity ratio of the peak and the ASE. The estimated intensity of the ASE was $\sim 4 \times 10^{9} \mathrm{~W} \mathrm{~cm}^{-2}$.

The conical target was made using laser machining of aluminium foils of various thicknesses. First, as shown in figure 1(a), an Al foil was set so that the tip thickness of the conical target is equal to the desired value: $d$. Note that the focus of the laser is set at the bottom of the conical targets regardless of its $d$. Second, a preset number of pulses (machining pulse) from the regenerative amplifier were focused onto the foil. The pulse energy was varied by the use of a half waveplate or changing beam diameter so the axis of the beam remains intact. As a consequence, the $f$-number of the machining pulse was changed. In order to keep reasonable machining time, we used $F / 6$ machining pulses with $\sim 7 \mu \mathrm{J}$ for initial foil thickness: $L=25 \mu \mathrm{m}$ but $F / 2$ machining pulses with $\sim 70 \mu \mathrm{J}$ for $L=50$ and $100 \mu \mathrm{m}$. Conditions of the machining process and its precision are summarized in table 1. The target will be referred to by the 'label' shown in the first column in table 1 i.e. conical target $\{\mathrm{A}\}$. The preset number could be changed in order to control the thickness of the tip of the conical target. The preset number is given by $(L-d) / l_{\mathrm{s}}$, where $L$ and $l_{\mathrm{s}}$ are the initial foil thickness and the thickness removed by a single machining pulse, respectively. $l_{\mathrm{s}}$ is given by $L / N_{\mathrm{D}}$, where $N_{\mathrm{D}}$ is the number of machining laser pulses necessary to penetrate through the foil. The penetration was confirmed by measuring transmittance of a HeNe laser beam, which is sent along to the laser axis. The transmitted HeNe light was scattered by diagnostics positioned behind the target, which are described below (LANEX screen and CR39 film) and was detected by a CCD (charged coupled device) camera.

The camera images the diagnostic surface. $l_{\mathrm{s}}$ was measured before each individual shot sequence for machined conical targets. Finally, a $30 \mathrm{TW}$ pulse irradiated the conical target without alignment, since the focal position and the laser axis were observed to be identical with respect to the machining pulse and the high intensity $30 \mathrm{TW}$ pulse: figure $1(b)$.

The scanning electron microscope (SEM) image observing the front and the rear surface of the conical target is shown in figures $1(c)$ and $(d)$, respectively. Here we refer to the 'rear surface' as the surface not illuminated by the high intensity laser and the 'front surface' for the laser illuminated surface. The hole radius on the front surface was $\sim 5 \mu \mathrm{m}$ and the wall surface in the interior of the cone had a roughness of about $1 \mu \mathrm{m}$. We found that a larger interior structure is observed when 10 times higher machining pulse energy was used as shown in figure $1(e)$. This difference could be utilized for controlling the interior roughness by tuning machining pulse energy after proper parameter studies. The roughness of the interior surface may enhance laser absorption of the laser beam in the experiments with larger beam divergence.

A conical shape is clear in figures $1(f)$ and $(g)$ in which front hole radius is plotted as a function of the distance from the focus. Here the distance from the focus was varied by changing $L$. A similar plot is obtained for vertical radius but the radius is slightly smaller due to oblique incidence. Radius is consistent with a Gaussian beam waist at the given $f$-number. Linear fitting reproduces data points better than the radius of the Gaussian beam, as shown by dashed-dotted lines in figures $1(f)$ and $(g)$. Table 2 summarizes the cone target parameters obtained from the fitting curves.

The front hole must be larger than the spot size of the laser in order to avoid energy loss due to clipping of the laser beam. Figures $1(f)$ and $(g)$ show that the front hole is larger than the beam waist of the $30 \mathrm{TW}$ pulse except for two data points, at $L=50 \mu \mathrm{m}$ and $100 \mu \mathrm{m}$. We did not use conical targets with these parameters for experiments, therefore the clipping should not be a problem for any hole depth dependence in the experiments.

A series of hydrodynamic simulations in a 1D planar geometry was performed in order to obtain insight into the preplasma density profile as shown in appendix A. A third order autocorrelator revealed two precursor pulses in the laser temporal profile: one is the ASE pulse and the other is a foot of the pulse. Idealized intensity profiles of the precursor pulses are shown in figure A1 of appendix A.

The intensity of the ASE pulse linearly ramps at $2 \mathrm{~ns}$ before the main pulse. The estimated intensity of the ASE was $\sim 4 \times 10^{9} \mathrm{~W} \mathrm{~cm}^{-2}$ and this intensity may be below the damage threshold. However, as described in section 4, the focusing effect due to the conical target might result in an ASE pulse 


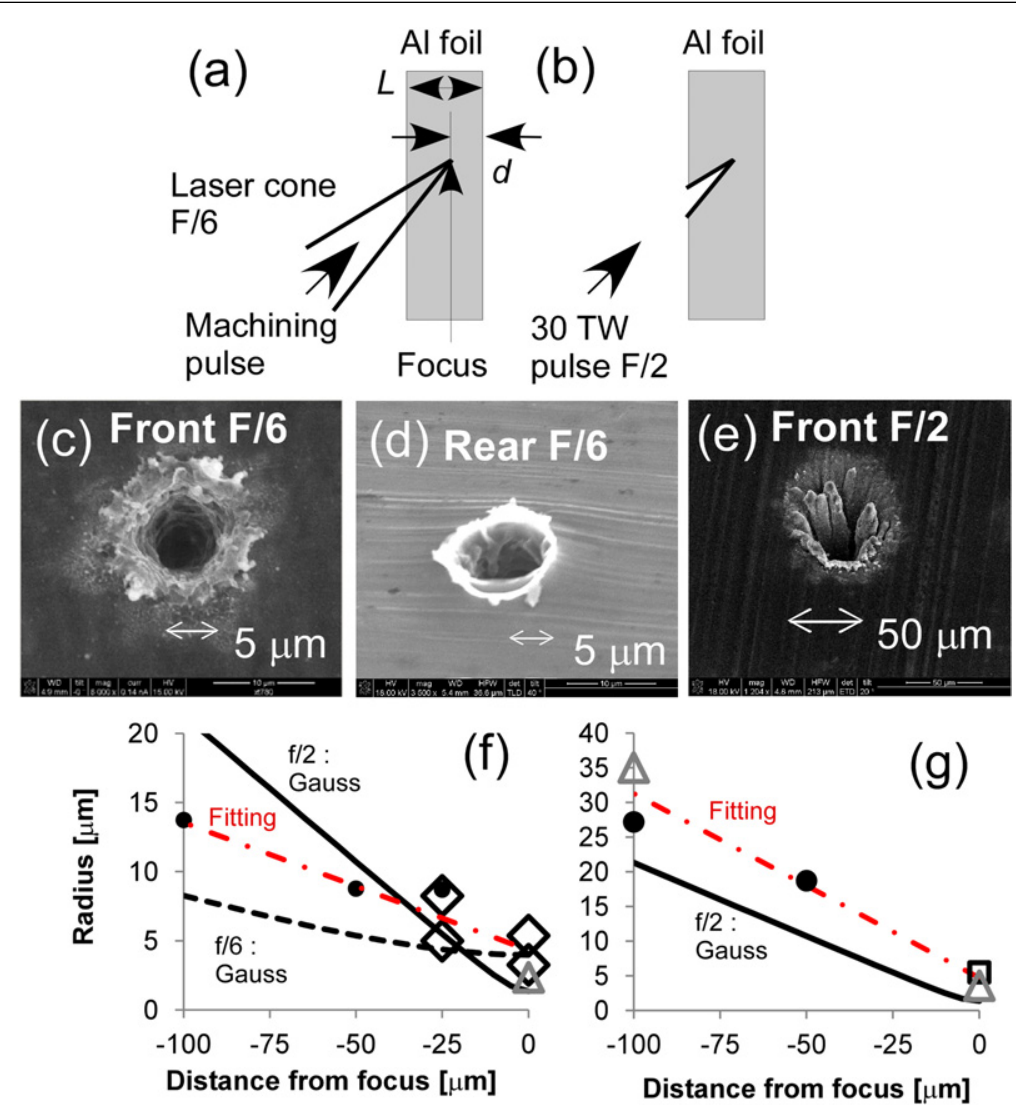

Figure 1. Conical target machining process $(a)$ pre-drilling geometry and $(b)$ interaction geometry at 30 TW laser power (note that the angle of incidence is exaggerated here-it was $20^{\circ}$ from normal during the experiments). SEM images of the hole after the machining process by $F / 6$ pulses $(c)$ the front and $(d)$ rear surface of the conical target $\{\mathrm{A}\}$. (e) SEM image of the front surface hole of the conical target $\{\mathrm{E}\}$ after the machining process by $F / 2$ pulses. Horizontal radius of the laser pulse and the hole radius of the conical target made by $(f) F / 6$ with $\sim 7 \mu \mathrm{J}$ and $(g) F / 2$ with $70 \mu \mathrm{J}$ machining pulse, respectively. Here, points of the diamonds: obtained from SEM images for $L=25 \mu \mathrm{m}$, the solid circles: from optical microscope objective images, triangles: obtained from SEM images for $L=100 \mu \mathrm{m}$. $L$ of optical microscope points is equal to 'distance from focus' in the figures. Beam waist radius of $F / 6$ and $F / 2$ Gaussian beam are shown by dashed and solid lines. Linear fitting line for data points are shown by dashed-dotted lines. Note that the beam waist values are divided by $\cos \left(20^{\circ}\right)$ in order to take into account tilt of the target.

Table 1. Condition of laser machining and parameters of conical targets. Values in parentheses indicate standard deviation (STDEV) which is used to estimate error for the tip thickness of conical targets.

\begin{tabular}{llllrl}
\hline & $\begin{array}{l}\text { Machining } \\
\text { pulse } \\
\text { energy } \\
(\mu \mathrm{J})\end{array}$ & $F / \#$ & $l_{\mathrm{s}}(\mu \mathrm{m} /$ shot $)$ & $\begin{array}{l}L \\
(\mu \mathrm{m})\end{array}$ & $\begin{array}{l}\text { Number of } \\
\text { samples }\end{array}$ \\
\hline $\mathrm{A}$ & 7 & $F / 6$ & $0.84(0.05)$ & 25 & 4 \\
$\mathrm{~B}$ & 7 & $F / 6$ & $0.91(0.1)$ & 50 & 2 \\
$\mathrm{C}$ & 7 & $F / 6$ & $0.15(0.03)$ & 100 & 2 \\
$\mathrm{D}$ & 70 & $F / 2$ & $2.3(0.2)$ & 50 & 3 \\
$\mathrm{E}$ & 70 & $F / 2$ & $1.6(0.1)$ & 100 & 2 \\
$\mathrm{~F}$ & 180 & $F / 2$ & 4.2 & 100 & 1 \\
\hline
\end{tabular}

with intensity on the order of $10^{10} \mathrm{~W} \mathrm{~cm}^{-2}$ and may produce some preplasma. For the foot of the pulse, intensity rapidly increases from $50 \mathrm{ps}$ before the main pulse and could reach a very high intensity. According to the results, plasma expansion velocity at relativistic critical density: $n_{\mathrm{cr}} \sim 10 n_{\mathrm{c}}$ for peak laser intensity is too small (expansion distance on the order of micrometre at the arrival of the main pulse) to fill up the conical hole in this experiment. Here relativistic critical density is
Table 2. Parameters of conical targets. 'Hor.' and 'Vert.' indicate values in the horizontal and the vertical directions. The vertical direction is perpendicular to the page in figure $1(a)$.

\begin{tabular}{lll}
\hline $\begin{array}{l}\text { Machining } \\
\text { configuration }\end{array}$ & $\begin{array}{l}\text { Total } \\
\text { opening angle }\left({ }^{\circ}\right)\end{array}$ & Tip diameter $(\mu \mathrm{m})$ \\
\hline$F / 6,7 \mu \mathrm{J} /$ pulse & Hor. 11, Vert. 8 & Hor. 4, Vert. 5 \\
$F / 2,70 \mu \mathrm{J} /$ pulse & Hor. 30, Vert. 22 & Hor. 5, Vert. 5 \\
\hline
\end{tabular}

given by $n_{\mathrm{cr}}=n_{\mathrm{c}} \gamma$ with the Lorentz factor, $\gamma=\sqrt{1+a_{0}^{2} / 2}$, and the critical density is $n_{\mathrm{c}}=1.11 \times 10^{21} / \lambda_{\mu}^{2}$ in unit of $\mathrm{cm}^{-3}$ [18-22]. However, lack of various collective absorption mechanisms of the laser to the plasma in the simulation code may cause the preplasma scale length to be underestimated. Thus the experimental results provide further insight for the preplasma scale length.

\section{Optimum hole depth of conical targets for hot electron beam production}

The laser irradiated targets at a $20^{\circ}$ incidence angle using $P$ polarization. Hot electron production was studied using 

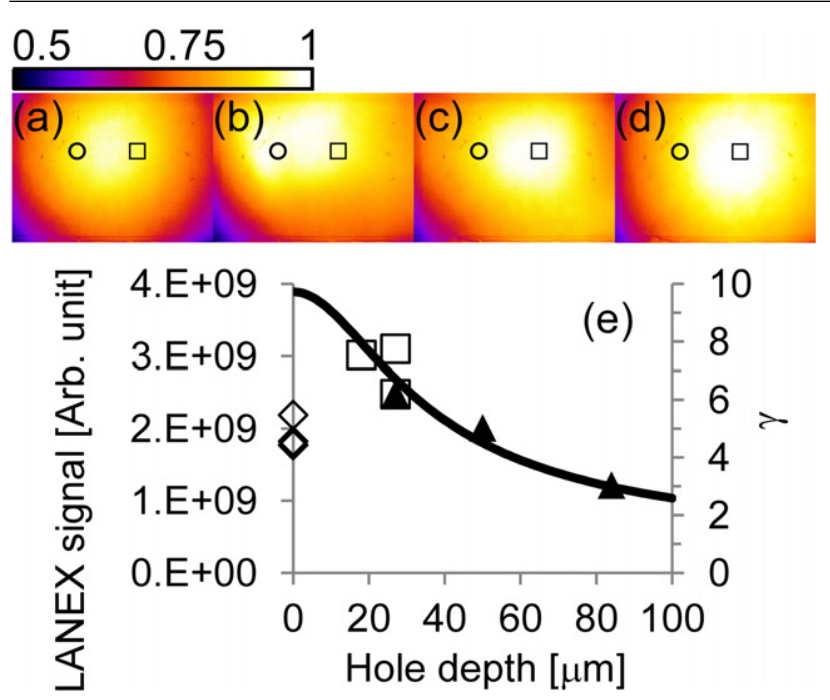

Figure 2. Hot electron beam profiles from $(a)$ the flat target $\mathrm{FWHM}=80^{\circ},(b)$ conical targets with hole depth of $27 \mu \mathrm{m}$ $\mathrm{FWHM}=100^{\circ},(c) 50 \mu \mathrm{m} \mathrm{FWHM}=90^{\circ}$ and $(d) 84 \mu \mathrm{m}$ $\mathrm{FWHM}=93^{\circ}$. Initial target thickness is fixed at $100 \mu \mathrm{m}$. The conical targets $\{\mathrm{E}\}$ were used for $(a)-(c)$ but $\{\mathrm{F}\}$ for $(d)$. The intensity is normalized by the peak intensity of each image. The circle and the square indicate the laser axis and the target normal direction, respectively. (e) Integrated signal counts from the CCD camera for the LANEX screen as a function of the hole depth from initial target thickness of $50 \mu \mathrm{m}$ with conical targets of $\{D\}$ (square) and $100 \mu \mathrm{m}$ with conical targets of $\{\mathrm{E}\}$ and $\{\mathrm{F}\}$ (triangle). The solid curve shows Lorentz factor corresponding to the laser intensity in vacuum at the entrance of the hole. Note that a conical target of $\{\mathrm{F}\}$ is only used for the data point at hole depth of $84 \mu \mathrm{m}$. The flat target is shown by diamonds and the thickness is 50 and $100 \mu \mathrm{m}$.

The error bar of the hole depth is approximately the same as the size of data points (not shown).

two diagnostics. The hot electron beam spatial profile was measured using a LANEX scintillation (KODAK) screen which was placed $7.5 \mathrm{~cm}$ behind the target. A $200 \mu \mathrm{m}$ thick Al filter was positioned in front of the screen to block lower energy electrons below $200 \mathrm{keV}$ as well as light emission. The rear side of the screen was imaged onto a CCD camera to observe light emission caused by electrons incident onto the screen. A $2 \mathrm{~mm}$ thick BG39 filter was used to remove scattered laser light. The hot electron beam charge was inferred by integrating signal counts on the CCD camera. The hot electron beam charge could also be determined by measuring the bremsstrahlung $\gamma$-ray yield resulting from the hot electron beams produced. $\gamma$-ray photons were detected using a $\mathrm{NaI}$ scintillator coupled to a photomultiplier which was positioned at $30^{\circ}$ above the laser axis. Low energy $\gamma$-ray photons below $1 \mathrm{MeV}$ energy were blocked using a 6 inch thick lead brick in front of the detector. The detector was surrounded by 2 inch of lead which blocks other scattered $\gamma$-rays. The integrated signal count from the LANEX screen images was found to be proportional to the $\gamma$-ray signal from the $\mathrm{NaI}$ scintillator.

Images of the hot electron beam profile are shown in figures $2(a)-(d)$ as a function of the hole depth for which the initial target thickness was fixed at $100 \mu \mathrm{m}$. Note that the conical targets $\{\mathrm{E}\}$ were used except for figure $2(d)$ which is obtained with the conical target $\{\mathrm{F}\}$.

Using flat targets, the direction of the hot electron beam was consistently positioned between the target normal direction and the laser axis (figure 2(a)). By using conical targets and increasing the hole depth, the peak of the beam shifts consistently with the laser axis (figure $2(b)$ ). Increasing the hole depth further, we observed the peak of the beam shifts to the target normal direction (figures $2(c)$ and $(d)$ ). The FWHM horizontal beam diameter varies between $80^{\circ}$ and $100^{\circ}$ and shows a maximum for the $27 \mu \mathrm{m}$ hole depth image. Since the FWHM beam diameter is larger than the LANEX screen, the beam divergence is obtained by fitting the intensity profile to a Gaussian function.

The hot electron beam charge was greatest at the $\sim 20 \mu \mathrm{m}$ hole depth as shown in figure 2(e). This depth matches well with the Rayleigh length of the laser pulse: $25 \mu \mathrm{m}$. We found that the shot averaged hot electron beam charge in a hole depth ranging between 18 and $27 \mu \mathrm{m}$ is $44( \pm 18) \%$ greater than for the flat target. We found no correlation between $\gamma$-ray signal and thickness for the flat target in the range between 0.8 and $100 \mu \mathrm{m}$. Therefore, it is clear the difference of the hot electron beam charge is due to the influence of the hole depth. The increase in the hot electron beam charge is comparable to the values reported in [3].

As shown in the literature $[10,11]$, preplasma generated by an ASE pulse could prohibit the propagation of the main laser pulse to the tip of the conical target due to absorption or scattering. The drop in the electron yield at the hole depth of 50 and $84 \mu \mathrm{m}$ could be the influence of the preplasma. Insight into the interaction may be obtained when hydrodynamic simulation results are considered. According to the simulations, the density point of $n_{\mathrm{c}}$ moves from the conical wall at a distance several times greater than that of $n_{\mathrm{cr}} \sim 10 n_{\mathrm{c}}$ (figure $\mathrm{A} 1(c)$ ). The difference is even larger when the distance is compared between $0.01 n_{\mathrm{c}}$ and $10 n_{\mathrm{c}}$. Note that in the literature $[10,11]$, experiments clearly showed that a preplasma of $0.01 n_{\mathrm{c}}$ density inhibits laser propagation to the tip of the conical target for vacuum focal laser intensity corresponding to $a_{0} \sim 3$. In our experiments, the focused laser intensity is much higher and $a_{0} \sim 14$ at vacuum focus. Such high laser intensity allows relativistically induced transparency (RIT) up to the relativistic critical density [18-22]. It might be reasonable to expect that the electron yield is proportional to the $n_{\mathrm{cr}}$ where the laser interacts with the plasma most strongly. In order to elucidate the influence of RIT to the electron yield, $\gamma$ is estimated using the laser intensity in vacuum at the entrance of the hole. Note that the focus of the laser is always at the bottom of the conical target regardless of $d$. The agreement between $\gamma$ and data points is remarkable despite this simple argument, as shown in figure $2(e)$. We also found that by using an argument of energy balance between the plasma and the laser pulse for RIT [22], the pump depletion length is found to be $l_{\mathrm{pd}}=2 c \tau n_{\mathrm{c}} / n_{\mathrm{e}}$ with $c$ as the speed of light. When we assume the conical target is filled by a $n_{\mathrm{e}}=0.02 n_{\mathrm{c}}$ density plasma, the equation yields $l_{\mathrm{pd}}=90 \mu \mathrm{m}$, which is approximately the same as the deepest hole in the experiments. Note that the density is well matched with the prediction of the hydrodynamic simulation as shown in figure $\mathrm{A} 1(c)$, which shows that the $0.01 n_{\mathrm{c}}$ density surface moves more than $10 \mu \mathrm{m}$ from the wall, and this distance is comparable to the diameter of the hole. Therefore, we conclude that preplasma would lower the electron yield since the underdense plasma inhibits laser propagation deep into the conical target. Note that, for 
(a)

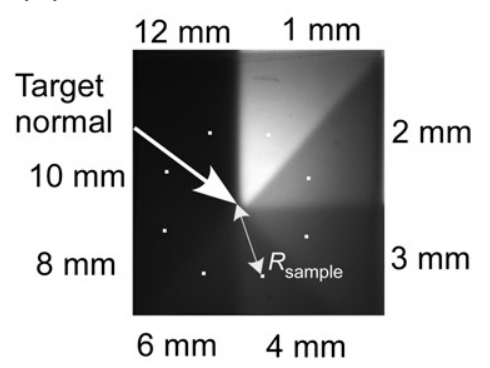

(b)

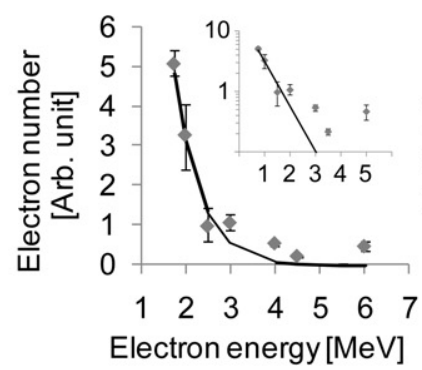

(c)

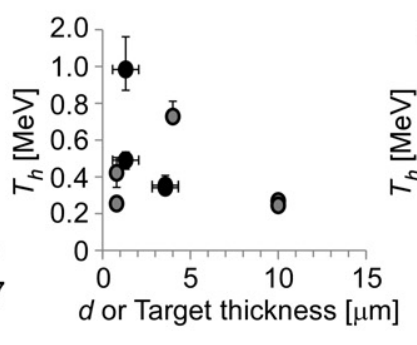

(d)

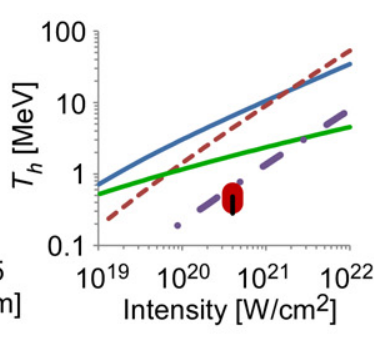

Figure 3. (a) LANEX screen image with Al filters, where the numbers indicate total Al filter thickness. Sampling region for signal integration is shown by white boxes on a circle at the radius of $R_{\text {sample }}$. $(b)$ Hot electron energy spectrum obtained from $(a)$. The inset is the same plot but the vertical axis is on logarithmic scale. Here, points are experimental data and the line is least squares fit using the Maxwellian distribution function which is given in the text. Error bars are for the data points when the sampling radius $\left(R_{\text {sample }}\right)$ was changed by $\pm 50 \%$ with sample size fixed. $(c) T_{\mathrm{h}}$ dependence on target thickness, where grey points are from flat targets and black points are from conical targets $\{\mathrm{A}\}$. Error bars are due to fitting uncertainty. $(d)$ Laser intensity dependence of $T_{\mathrm{h}}$. Curves are obtained from (solid blue line) $T_{\mathrm{p}}$ : ponderomotive scaling, (solid green line) equation (11) from [27], (dashed red line) $T_{\mathrm{mp}}$ : equation (2) from [25] with $n_{\mathrm{e}}=10 n_{\mathrm{c}}$ and (dashed-dotted purple line) from $T_{\mathrm{mp}}$ with $n_{\mathrm{e}}=450 n_{\mathrm{c}}$, which corresponds to solid density of aluminium. Range of experimental data points are shown as red points for conical targets and vertical black line for flat targets, respectively.

the short length conical hole, sizeable energy could reach the tip due to a combination of RIT and self-focusing of the laser beam [23]. The argument here could provide some remedy for the inhibition of the laser propagation by utilizing RIT.

In some experiments, a set of Al filters were placed in front of the LANEX screen in order to infer the hot electron energy spectrum. Wedge-shaped aluminium plates with eight different thicknesses were assembled so that the apex of each plate is placed in the normal direction of the target (figure 3(a)). The hot electron energy spectrum is therefore obtained by integrating signal counts in a small region as indicated by the white boxes in figure $3(a)$. The hot electron beam is not uniform as shown in figure 2 . We set the integration region on a circle with a radius of $R_{\text {sample }}$. We found that varying $R_{\text {sample }}$ by $\pm 50 \%$ does not change the electron spectrum and estimated the electron temperature, thus taking sampling point on a circle is reasonable. The electron energy $(\varepsilon)$ for each filter was given by the stopping range of known $\mathrm{Al}$ thicknesses. The hot electron energy spectrum was fitted by a Maxwell distribution function: $n_{\mathrm{e}}(\varepsilon) \propto \exp \left(-\varepsilon / T_{h}\right)$ in order to estimate the electron temperature (figure $4(b))$. The inferred temperature $\left(T_{\mathrm{h}}\right)$ for conical (flat) targets was in the range $0.2-1 \mathrm{MeV}(0.2-$ $0.8 \mathrm{MeV}$ ) for the range of target thicknesses as shown in figure $3(c)$.

The $T_{\mathrm{h}}$ is substantially lower than the predicted $T_{\mathrm{p}}=$ 6.5 MeV according to ponderomotive scaling [24], which is given by $T_{\mathrm{p}}=m c^{2}\left(\sqrt{1+a_{0}^{2}}-1\right)$ with $m$ being electron mass.

Lower temperatures could be explained by the interaction of the laser pulse with a sharp density gradient followed by a dense plasma with $n_{\mathrm{e}} \gg n_{\mathrm{c}}$, and the temperature scaling is then given by $T_{\mathrm{mp}}=m c^{2}\left(\sqrt{1+a_{0}^{2} / 2}-1\right)\left(n_{\mathrm{e}} / n_{\mathrm{cr}}\right)^{1 / 2}$, which showed an excellent agreement with PIC simulations [25]. For a large preplasma in which the scale length is larger than the plasma skin depth $\left(L \gg c / \omega_{\mathrm{p}}\right)$, laser absorption takes place at $n_{\mathrm{cr}}$ and $T_{\mathrm{mp}}$ reduces to $T_{\mathrm{p}}$. In this case, the full electron excursion length: $l_{\mathrm{ex}}=\left(a_{0}^{2} / 4 \gamma^{2}\right)\left(c / \omega_{0}\right)$ in the plane wave is realized [26]. For $a_{0} \gg 1$, the length is reduced to $l_{\mathrm{ex}} \sim \lambda / 4 \pi$. On the other hand, for a small scale length: $L<l_{\mathrm{ex}}$, the laser field could interact with a plasma whose density is much larger than $n_{\mathrm{cr}}$. Indeed, the $n_{\mathrm{cr}}$ density point is within a fraction of wavelength for flat targets (appendix A). As a consequence, $l_{\mathrm{ex}}$ is reduced since electrons dragged out from the dense plasma shield the laser field, which therefore lowers temperature. We found that $T_{\mathrm{mp}}$ shows good agreement with experimental data points when vacuum focal intensity and solid Al plasma density: $450 n_{\mathrm{c}}$ are used (figure $3(d)$ ).

This lowering of the hot electron temperature by reducing the preplasma scale length may be useful for FI where electron beams of a few megaelectronvolts are optimal for fuel core heating. Slightly higher shot averaged $T_{\mathrm{h}}$ is observed for conical $(0.54 \pm 0.2 \mathrm{MeV})$ target than flat targets $(0.38 \pm$ $0.2 \mathrm{MeV}$ ). This could be interpreted as larger preplasma scale length due to intensity enhancement of the ASE pulse as described in the next section. We found that the temperature for the conical target at $d \sim 1 \mu \mathrm{m}$ fluctuated shot to shot much more than $d \sim 4 \mu \mathrm{m}$, while the flat target shows relatively small shot to shot fluctuation. This could be because absorption depends strongly on shot to shot variations in the target conditions possibly due to the ASE content and intensity in the laser pulse. Therefore, understanding the influence due to precursor pulses on the preplasma density scale length is critical for designing of fast ignitors. We note that the fluctuation could be due to the fluctuation of the conical target shape.

\section{Maximum proton energy from flat and conical targets}

In this experiment the initial target thickness for conical targets was set at $25 \mu \mathrm{m}$ and $d$ was varied. A CR39 track detector was set $6 \mathrm{~cm}$ behind the target and each CR39 was exposed for 2-6 shots. Various thicknesses of Mylar filters were placed in front of the CR39 in order to obtain the beam profile for different proton energies. A $12 \mu \mathrm{m}$ Al filter covered the entire CR39 to block laser light. The thickness of the Mylar filter was varied spatially as shown in figure $4(a)$. The maximum proton energy is inferred by the stopping range of the proton for the thickest filter where proton tracks were observed. In some shots, proton tracks are missing for thick Mylar filters as shown 
(a)
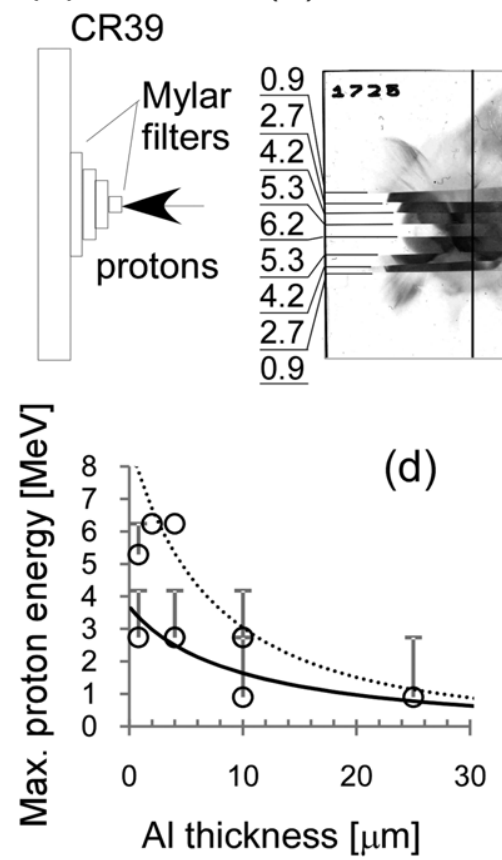

(b)

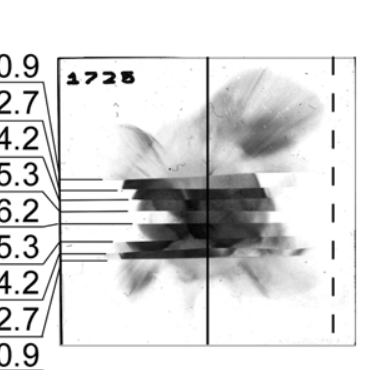

(c)

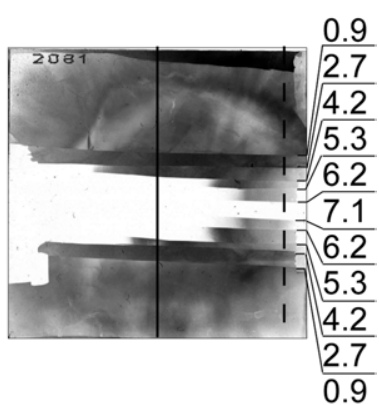

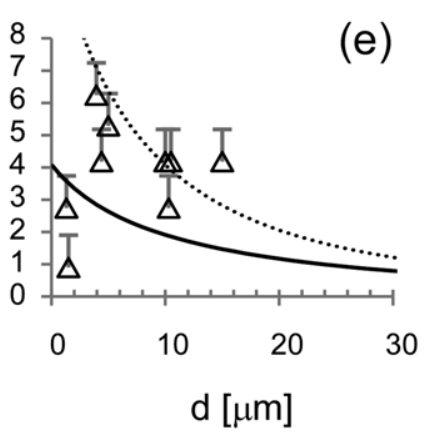

(e) 西}

(1)


duration. The acceleration time is taken to be $1.3 \tau$, which represents saturation time of proton energy observed in 2DPIC simulations in [32] for a wide range of laser intensities and pulse durations. The electron density is estimated by the equation: $n_{\mathrm{e} 0}=N_{\mathrm{e}} /\left(c \tau S_{\text {sheath }}\right)$ where $N_{\mathrm{e}}=f E / T_{\mathrm{h}}$ is the total number of electrons in the beam and $S_{\text {sheath }}=$ $\pi\left(r_{0}+\delta \tan (\theta)\right)^{2}$ is the hot electron beam cross sectional area at the rear surface for a target thickness of $\delta$. We used experimentally obtained values for $T_{\mathrm{h}}$. The absorption fraction, $f$, is assumed to be 0.5 for flat targets and 0.7 for conical targets. The difference in $f$ is taken from hot electron beam charge measurement results. Here, $r_{0}$ is the laser spot radius and $\theta$ is the half angle of the hot electron beam divergence. We used $\theta$ as a free parameter to obtain the best fit $\left(\theta=15^{\circ}\right)$ for flat targets.

For flat targets, equation (1) reproduces the experimental data points well as shown in figure $4(d)$. For conical targets, equation (1) reproduces experimental data points in $d=4 \mu \mathrm{m}$ and $10 \mu \mathrm{m}$ but overestimates the proton energy for $d<4 \mu \mathrm{m}$. This discrepancy indicates that the sheath field is decreased by a plasma expansion preceding the main pulse due to the ASE pulse. The fact that the proton beam shift was observed for $d \leqslant 4 \mu \mathrm{m}$ also supports this interpretation.

Note that the influence of the ASE pulse on TNSA could be negligible, in particular to the maximum proton energy up to some thickness where the proton energy starts to fall [33]. We found that the $10^{10} \mathrm{~W} \mathrm{~cm}^{-2}$ ASE could produce plasma at the rear surface and the plasma expansion is estimated to be less than a micrometre for a $2 \mu \mathrm{m}$ tip thickness (appendix A) but no plasma expansion is observed when the ASE pulse at $10^{9} \mathrm{~W} \mathrm{~cm}^{-2}$ was used. Despite lacking several aspects of the interaction physics in the simulation code, we believe that the discrepancy between conical and flat targets is due to intensity enhancement of the ASE pulse due to conical targets. This belief is reinforced by experiments that have been performed at the Lund Laser Center in Sweden [34]. The experiments clearly demonstrated that an external laser pulse, synchronized to an ultra-intense laser pulse for proton acceleration from a $3 \mu \mathrm{m} \mathrm{Al}$ foil, were responsible for the proton beam shift from the target normal direction and not ceasing proton acceleration. The external laser pulse parameters were $3 \times 10^{10} \mathrm{~W} \mathrm{~cm}^{-2}$ at $l=532 \mathrm{~nm}$ with duration variation between 3 and $12 \mathrm{~ns}$ and very similar to our ASE pulse intensity. Therefore, we conclude the presence of ASE pulse at the order of $10^{10} \mathrm{~W} \mathrm{~cm}^{-2}$ would not inhibit TNSA but may still influence the proton beam direction.

It is interesting to note that using the same value of $\theta$ for flat targets with equation (1) reproduces experimental results for conical targets. The hot electron beam from the conical targets has a $25 \%$ larger divergence angle than from flat targets, as shown in figure 2 . Equation (1) with $\theta=19^{\circ}$ does not change the curve significantly. Despite this fact, $\theta$ could influence maximum proton energy for the following two reasons. First, the divergence angle measurements could be affected by a selfgenerated field at the rear side of the target including a sheath field and an azimuthal magnetic field which could alter intrinsic beam divergence upon escaping the target [35]. Second, $\theta$ could be a function of $d$ since the interaction with the preplasma and the wall of the conical target might increase $\theta$. These two effects make the estimation of $\theta$ difficult.
The maximum proton energy is similar for flat and conical targets, even though there is increased hot electron beam charge for conical targets. This may be understood by considering the difference in the hot electron beam divergence as described above. We note at the end that hot electron temperature is the most sensitive parameter according to equation (1) where the proton maximum energy depends linearly on $T_{\mathrm{h}}$ but depends on hot electron beam density logarithmically. In our experiments, $T_{\mathrm{h}}$ was found to be similar between flat and conical targets.

\section{2D-PIC simulations}

2D-PIC simulations were performed for a flat and a conical target in order to understand electron and proton beam acceleration. To achieve good computational accuracy and avoid self-heating in the simulation due to the interaction of the laser pulse with solid density plasma, we performed simulations with higher laser intensity and a lower ionization state of target as compared with the experimental parameters. With this approach we aim to identify the same qualitative trends in electron and ion acceleration as described above for experimental conditions without a detailed quantitative comparison. The initial target thickness was $15 \lambda(2 \lambda)$ and the transverse width was $10 \lambda$ for the conical (flat) target. The tip diameter of the conical hole was $\lambda$ and the front hole diameter was $5 \lambda$. The conical tip thickness was $2 \lambda$. Fully ionized Be was assumed for the target at an electron density of $n_{\mathrm{e}}=160 n_{\mathrm{c}}$. A $50 \mathrm{~nm}$ proton layer was attached on the rear surface of the target in order to simulate a contamination layer. A preplasma with a $2 \lambda$ linear density ramp was placed near the tip of the cone to model a finite ASE intensity contrast of the laser pulse. A 35 fs FWHM laser pulse at peak normalized vector potential of $a_{0}=50$ irradiated the conical target at a normal angle of incidence. A linearly polarized pulse was used such that the electric field of the laser lies in the $z$ direction, which is perpendicular to the images shown in figures 5 and 6 .

The contour image in figures $5(a)$ and $(b)$ of the electron density shows more beam spread of hot electrons for the conical target than for the flat target which is consistent with experimental observations. The hot electron energy spectrum as a function of the transverse coordinate from the flat and the conical target is shown in figures $5(c)$ and $(d)$, respectively. Larger hot electron beam divergence is clearly shown for the conical target than for the flat target. For the conical target, the hot electron beam is not only generated at the tip of the conical target but also on the conical wall surface and the latter beam increases the beam divergence. The total number of hot electrons was at least two times larger than from the flat target, but the hot electron temperature was approximately the same in both cases. This qualitatively agrees with the experimental observation of the electron beam.

Despite the difference in $T_{\mathrm{h}}$ between the experiments $(\sim \mathrm{MeV})$ and the simulations $(\sim 20 \mathrm{MeV})$, we believe that the simulation is relevant for our experiments since the interaction is ultra-relativistic for both cases, therefore capturing the essential aspects of the interaction.

We note here that the influence of the divergence angle and the energy spectrum of the electron beam on the proton acceleration due to TNSA requires a detailed analysis of the electrical sheath structure and its correlation between the 

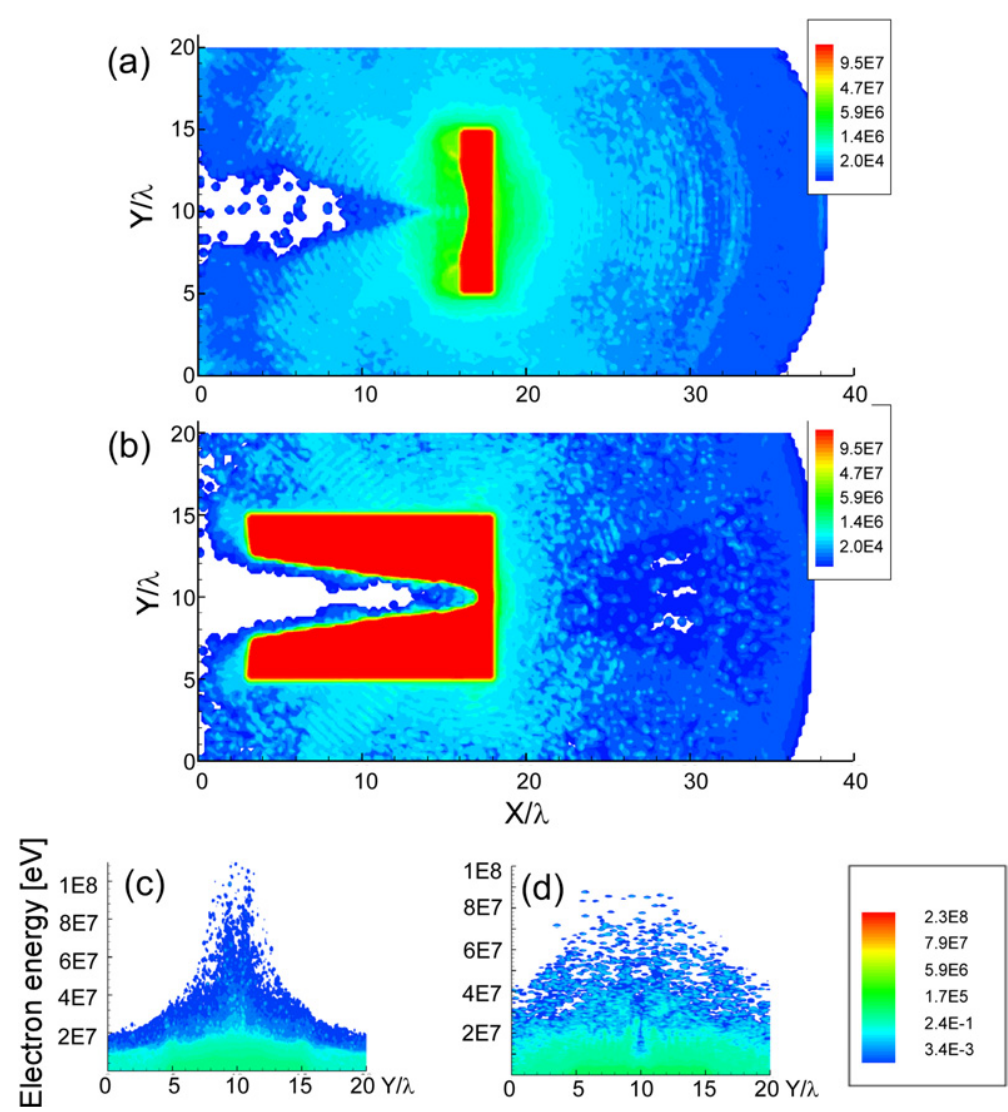

Figure 5. Simulated electron density contour for $(a)$ the flat target and $(b)$ the conical target. The laser pulse irradiates the targets from left boundary along the $X$ direction. Hot electron energy distribution from 2D-PIC simulations for $(c)$ a flat target and for $(d)$ a conical target. The vertical axis is electron energy and horizontal axis is spatial axis in the $Y$ direction. The colour scale represents the electron density.

(a)

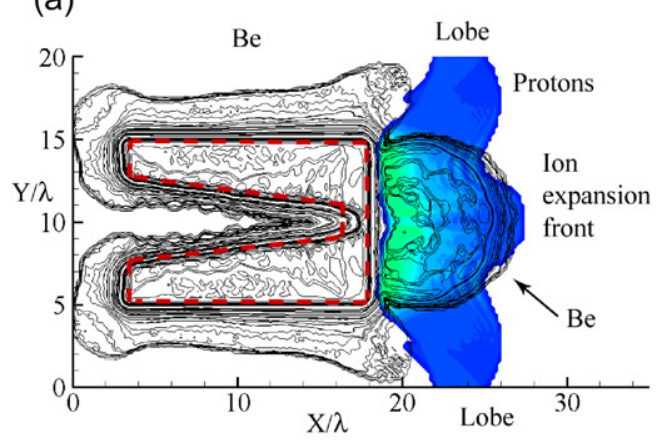

(b)

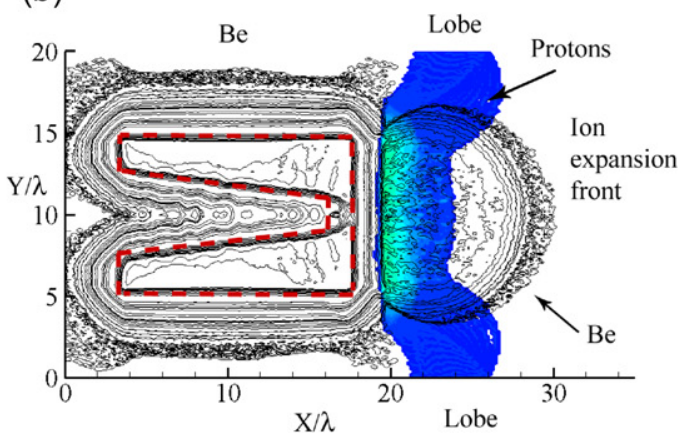

(c)

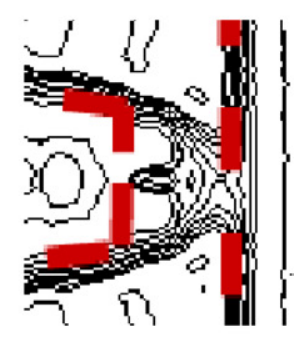

Figure 6. Ion density contour from 2D-PIC simulations for the conical target ( $a$ ) with preplasma at $t=200 \mathrm{fs}$ and $(b)$ without preplasma at $t=230$ fs. (c) Zoom up image of the tip of the conical target from $(b)$. Dashed lines in the figures show initial target surface. The laser pulse irradiates the target from the left boundary normal to the target.

sheath field and the property of electron beams. This influence is critical to FI and more work is needed.

We also observed heavy ion acceleration which is represented by figure 6 for two cases: with and without preplasma. The second case corresponds to high intensity contrast ratio that could be relevant to future experiments. In the case of preplasma, which is more relevant to our experiment, $\mathrm{Be}^{4+}$ ions surprisingly gained almost the same energy ( $\sim 20 \mathrm{MeV} /$ nuclei) as the protons $(\sim 20 \mathrm{MeV})$ so that proton and $\mathrm{Be}^{4+}$ fronts move with approximately the same velocity. This is nontrivial and different from ion acceleration from flat targets. Approximately in the middle of the conical target, the spot diameter of the laser pulse is comparable to the hole diameter. This gives a complicated structure in the electromagnetic field near the bottom of the hole, which results in stochastic electron heating. Consequently, electron directionality (forward) is significantly destroyed and leads to a charge separation electrostatic field near the bottom, which is stronger than the rear surface field (TNSA field). Therefore, the Be ions from the hole are effectively accelerated in spite of a low $Z / A$ ratio and gain more energy compared with the case of the flat target.

In the simulation for the conical target without preplasma layer, $\mathrm{Be}^{4+}$ ions gained more energy $(21 \mathrm{MeV} /$ nuclei) than the 
protons $(\sim 11 \mathrm{MeV})$. Two 'lobes' of proton beams are observed which originate from the corner of the conical target. Those proton beams may be produced from the concentration of the electric field at the corner of the target which then produces a strong quasi-static electrical field for proton acceleration. In an actual target, the dimensions of the transverse size of the flat part of the target are on the order of millimetres and much larger than the simulated domain, hence such a field concentration cannot be expected. Thus, we neglect the proton beam component in the 'lobes' for this discussion. The higher Be ion energy suggests that the acceleration process is different from that for protons. The Be ion beam front shows a spherical shape with a diameter of $\sim 6.7 \lambda$ whose centre is shifted in the $X$ direction by $x_{\mathrm{c}} \sim 7 \lambda$. This ion front shape is different from that of protons, which show a flatter shape in the vicinity of the laser axis. In the simulation, protons were only located on the rear surface of the target, therefore, protons are only accelerated by the sheath field at the rear surface. On the other hand, the $\mathrm{Be}$ ions could be accelerated from the tip of the conical target. Our interpretation for the Be ion acceleration is the following. First, the ponderomotive force of the laser pulse pushes all electrons into the tip of the conical target. At the same time, the ions in the tip are also pushed by the ponderomotive force towards the laser axis with hole boring velocity $v_{\mathrm{hb}}=c \sqrt{\left(n_{\mathrm{c}} / 2 n_{\mathrm{e}}\right)(Z m / M)\left(I \lambda_{\mu}^{2} / 1.37 \times 10^{18}\right)}$ [24] during the laser pulse. Here, $Z$ and $M$ are ion charge state and ion mass, respectively. The laser intensity $(I)$ is given in units of $\mathrm{W} \mathrm{cm}^{-2}$. This equation gives $v_{\mathrm{hb}} \sim 0.043 \mathrm{c}$ and is consistent with velocity of the centre of the sphere observed in the simulation: $v_{\mathrm{c}} \sim x_{\mathrm{c}} / t=0.081 c$. Here, $t$ is the time in figure 6 and is measured from when the peak of the laser pulse arrives at the bottom of the conical target. In the frame moving at a velocity of $v_{\mathrm{c}}$, ions are accelerated by a symmetric electrical field arising from charge separation (Coulomb explosion) and are hence accelerated radially. As a consequence of hole boring and the Coulomb explosion, a spherical ion front whose centre is shifted away from the target normal is formed. This process is similar to the so-called 'directed Coulomb explosion' [36]. Actually, figure 6 shows an ion density depletion at the tip, which may be due to a Coulomb explosion. We found that the maximum $\mathrm{Be}^{4+}$ ion energy from the flat target is half that from the conical target. This might also suggest that the laser focusing in the conical shape [9] increases laser intensity compared with the flat target and yields a higher ion energy.

\section{Summary}

We have performed the first measurements of energetic electron and proton beams resulting from high intensity laser interactions with conical targets using laser machined targets. These 'proof of principle' experiments clearly demonstrate that with the use of this technique it is possible to perform systematic experiments, which can address much of the underlying physics of these complex targets-and can be directly compared with advanced simulations.

The results obtained by the experiments described here have some implications for the FI scheme where an ultraintense laser pulse irradiates normal to a conical target surrounded by a dense fuel plasma. In an actual FI scheme, the tip of the conical target is normal to the laser axis. The shift of the relativistic electron beam or the proton beam observed in the experiments would correspond to an increase in the divergence angle of the beam which reduces heating/coupling efficiency to the fuel core. We found that the shift of the proton beam direction was increased due to surface deformation, which is most likely induced by the ASE pulse whose intensity was increased by the conical shape. This result suggests that reducing the ASE intensity for conical targets could reduce proton beam divergence and can be beneficial for FI by the use of the proton beam as a heating source. We note here that the machining process could cause target rear surface deformation. Further technological development for monitoring morphology of the surface is desired. Reducing the prepulse may also be beneficial in that there is evidence from these experiments that this will cause a reduction in the generated electron temperature. 2D-PIC simulations confirmed these experimental results and further suggested that heavy ion acceleration in such experiments would be due to a 'directed Coulomb explosion,' which might be useful for accelerating heavy ions efficiently for medical or fusion applications.

\section{Acknowledgment}

This work was supported by the National Science Foundation through the Physics Frontier Center FOCUS (Grant No PHY0114336), the Russian Foundation for Basic Research and the International Science and Technology Center (Project No 2289). The SEM images were taken using the NOVA FIB in the Electron Microbeam Analysis Laboratory (EMAL) facility at the University of Michigan. This instrument was acquired with support from NSF grant \#DMR-0320740.

\section{Appendix A. 1D Hydrodynamics simulation for preplasma}

Hydrodynamic simulations using code 'HYADES' [37] were performed in order to study the preplasma density profile for our laser irradiation condition. To obtain a general idea, 1D planar geometry was used due to increased calculation speed. Laser absorption was taken into account by solving the Helmholtz equation for $P$ polarization at a normal incidence angle. Al was used as the target material. The electron density is obtained by assuming perfect ionization: $n_{\mathrm{e}}=13 \times n_{\mathrm{i}}$, where $n_{\mathrm{i}}$ is ion density since the peak intensity of the laser is comparable to the appearance intensity of $\mathrm{Al}^{13+}: 6.7 \times$ $10^{20} \mathrm{~W} \mathrm{~cm}^{-2}$ based on the barrier suppression ionization model [38]. Note that ionization potential for $\mathrm{Al}$ is obtained from web-site http://www.webelements.com/. Figure A1 $(a)$ shows the assumed laser intensity temporal profile, which reproduces our experimental measurement via third order autocorrelator [17] and consists of a $2 \mathrm{~ns}$ duration flat part (ASE pulse) and a rapidly rising part with a duration of $50 \mathrm{ps}$ : (foot pulses). The ASE intensity was set at $10^{10} \mathrm{~W} \mathrm{~cm}^{-2}$.

Two distinct features are observed due to the influence of ASE and foot pulses to the bulk of the target as shown in figure $\mathrm{A} 1(b)$ at the time when the main pulse arrives. First, a sharp shock in the bulk is observed due to the foot pulse. However, the relatively flat density profile is obtained when only the ASE pulse is irradiated. Second, when only the 

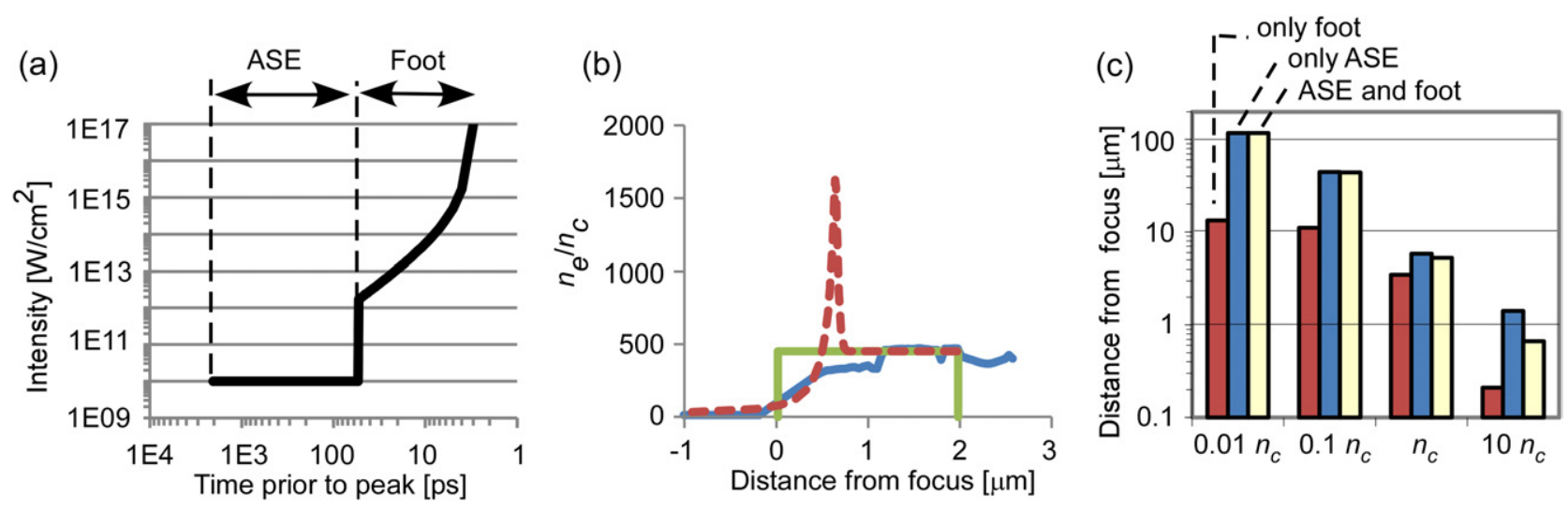

Figure A1. (a) Temporal pulse shape used in the hydrodynamics simulation. (b) Plasma density profile is shown as a red dashed (blue solid) line when only foot pulse is irradiated (only ASE pulse). The green rectangle shape curve shows initial density profile which is solid Al target with $2 \mu \mathrm{m}$ thickness. The laser pulse is irradiated from the left of the figure. (c) Distance from the initial front surface for various density points. The bars represent from left to right as only foot pulse, only ASE pulse and both pulses for simulations.

foot pulse is irradiated, no plasma was observed at the rear surface. However, when only ASE irradiates the target, plasma expansion at the rear surface on the order of a micrometre is observed. When both pulses are combined, peak density of the shock is reduced to $1000 n_{\mathrm{c}}$ and the location is shifted to $x \sim 1 \mu \mathrm{m}$ when the main pulse arrives. No other difference is observed. This could be explained via enhanced absorption of the laser due to longer scale length of the plasma as shown in figure $\mathrm{A} 1(c)$. As expected, the front surface plasma expands more for the ASE pulse irradiation than for the foot pulse irradiation. The distance between density point for $n_{\mathrm{c}}$ and the initial target surface is less than $10 \mu \mathrm{m}$ which is smaller than the dimension of the conical targets. Moreover, the distance between the initial target surface and the relativistic critical density is only a few micrometres. Here the relativistic critical density is $\sim 10 n_{\mathrm{c}}$ by using the intensity at vacuum focus for the peak of the main pulse.

Knowing the influence of incidence angle and polarization on the preplasma density profile is useful since the laser could be incident at a variety of angles and polarizations, especially at the wall. We found that using $S$ polarization or different incidence angles $\left(45^{\circ}, 65^{\circ}\right)$ does not change results significantly. This could be due to lack of collective effects in the simulation as described below. More realistic simulations, such as a 3D Hybrid simulation which can include nanosecond pulses and picosecond pulses in the same calculation, must be performed in order to predict experiments precisely. In 3D geometry, in which the plasma fills the hole, the density would be greater than in the $1 \mathrm{D}$ simulation.

In the simulation, collective effects such as vacuum heating processes [39] are not taken into account. Therefore, it should be noted that direct comparison between the experiment and those simulations might be problematic.

\section{References}

[1] Kodama R. et al 2001 Nature 412798

[2] Kodama R. et al 2004 Nature 4321005

[3] Chen Z.L., Kodama R., Nakatsutsumi M., Nakamura H., Tampo M., Tanaka K.A., Toyama Y., Tsutsumi T. and Yabuuchi T. 2005 Phys. Rev. E 71036403

[4] Flippo K.A. et al 2008 Phys. Plasmas 15056709
[5] Bulanov S.V. and Khoroshkov V.S. 2002 Plasma Phys. Rep. 28453

[6] Bulanov S.S. et al 2008 Med. Phys. 351770

[7] Cho B.I., Dyer G.M., Kneip S., Pikuz S., Symes D.R., Bernstein A.C., Sentoku Y., Renard-Le Galloudec N., Cowan T.E. and Ditmire T. 2008 Phys. Plasmas 15052701

[8] Nakatsutsumi M. et al 2007 Phys. Plasmas 14050701

[9] Sentoku Y., Mima K., Ruhl H., Toyama Y., Kodama R. and Cowan T.E. 2004 Phys. Plasmas 113083

[10] Baton S.D. et al 2008 Phys. Plasmas 15042706

[11] Rassuchine J. et al 2009 Phys. Rev. E 79036408

[12] Galloudec N.R., Cho B., Osterholz J. and Ditmire T. 2008 Rev. Sci. Instrum. 79083506

[13] Nakamura H.et al 2009 Phys. Rev. Lett. 102045009

[14] Nakamura T., Sakagami H., Johzaki T., Nagatomo H., Mima K. and Koga J. 2007 Phys. Plasmas 14103105

[15] Yanovsky V. et al 2008 Opt. Express 162109

[16] Bahk S.-W., Rousseau P., Planchon T.A., Chvykov V., Kalintchenko G., Maksimchuk A., Mourou G.A. and Yanovsky V. 2004 Opt. Lett. 292837

[17] Chvykov V., Rousseau P., Reed S., Kalinchenko G. and Yanovsky V. 2006 Opt. Lett. 311456

[18] Akheizer A.I. and Polovin R.V. 1956 Sov. Phys._JETP 3696

[19] Lefebvre E. and Bonnaud G. 1995 Phys. Rev. Lett. 742002

[20] Guerin S., Mora P., Adam J.C., Heron A. and Laval G. 1996 Phys. Plasmas 32693

[21] Fuchs J. et al 1998 Phys. Rev. Lett. 802326

[22] Willingale L. et al 2009 Phys. Rev. Lett. 102125002

[23] Matsuoka T. et al 2008 Plasma Phys. Control. Fusion 50105011

[24] Wilks S.C., Kruer W.L., Tabak M. and Langdon A.B. 1992 Phys. Rev. Lett. 691383

[25] Chrisman B., Sentoku Y. and Kemp A.J. 2008 Phys. Plasmas 15056309

[26] Landau L.D. and Lifshitz E.M. 1975 The Classical Theory of Fields (Oxford: Pergamon) p 118

[27] Haines M.G., Wei M.S., Beg F.N. and Stephens R.B. 2009 Phys. Rev. Lett. 102045008

[28] Wilks S.C., Langdon A.B., Cowan T.E., Roth M., Singh M., Hatchett S., Key M.H., Pennington D., MacKinnon A. and Snavely R.A. 2001 Phys. Plasmas 8542

[29] Lindau F., Lundh O., Persson A., McKenna P., Osvay K., Batani D. and Wahlstrom C.-G. 2005 Phys. Rev. Lett. 95175002

[30] Lundh O., Lindau F., Persson A., Wahlström C.-G., McKenna P. and Batani D. 2007 Phys. Rev. E 76026404

[31] Mora P. 2003 Phys. Rev. Lett. 90185002

[32] Fuchs J et al 2006 Nature Phys. 248 
[33] Kaluza M., Schreiber J., Santala M.I.K., Tsakiris G.D., Eidmann K., Meyer-ter-Vehn J. and Witte K.J. 2004 Phys. Rev. Lett. 93045003

[34] Lundh O., Glinec Y., Homann C., Lindau F., Persson A., Wahlström C.-G., Carroll D.C. and McKenna P. 2008 Appl. Phys. Lett. 92011504

[35] Yabuuchi T. et al 2007 Phys. Plasmas 14040706
[36] Bulanov S.S. et al 2008 Phys. Rev. E 78026412

[37] Larsen Jon T. and Lane Stephen M. 1994 J. Quant. Spectrosc. Radiat. Transf. 51179

[38] Augst S., Strickland D., Meyerhofer D.D., Chin S.L. and Eberly J.H. 1989 Phys. Rev. Lett. 632212

[39] Wilks Scott C. and Kruer William L. 1997 IEEE J. Quantum Electron. 331954 